\title{
Quantitative estimation of biological cell surface receptors by segmenting conventional fluorescence microscopy images
}

\author{
Julien Ghaye*, Chiara Succa*†, Danilo Demarchi ${ }^{\dagger}$, Sinan K. Muldur ${ }^{\ddagger}$, Pascal Colpo ${ }^{\ddagger}$, Paolo Silacci ${ }^{\S}$, \\ Guy Vergères ${ }^{\S}$, Giovanni De Micheli* and Sandro Carrara* \\ name.surname@ $\left\{\right.$ epfl.ch ${ }^{*}$, polito.it ${ }^{\dagger}$, jrc.ec.europa.eu ${ }^{\ddagger}$, alp.admin.ch $\left.{ }^{\S}\right\}$ \\ *Integrated Systems Laboratory, École Polytechnique Fédérale de Lausanne, EPFL, Lausanne, Switzerland \\ ${ }^{\dagger}$ Electronics and Telecommunication Department, Politecnico di Torino, Torino, Italy. \\ $\ddagger$ European Commission, Joint Research Centre, Institute for Health and Consumer Protection, Ispra, Italy \\ ${ }^{\S}$ Federal Department of Economic Affairs Education and Research EAER, Agroscope, Bern, Switzerland
}

\begin{abstract}
State-of-the-art techniques for measuring and monitoring gene level expression rely on messenger RNA (mRNA) extraction and quantification, usually based on the concept of reverse transcription polymerase chain reaction. In this paper, we take advantage of capabilities of image segmentation algorithms for monitoring target cell surface biomarkers using immunofluorescence microscopy. As a case study, we are looking at the expression level of toll-like receptor 2 (TLR2) proteins on Caco-2 intestinal cells after stimulation with lipopolysaccharide. The goal of this paper is to identify the segmentation algorithm which provides the best correlation between the pixel intensities of fluorescent images and quantified TLR2 mRNA. Three image segmentation algorithms are considered in this study for processing the fluorescent images acquired using a low-cost CMOS sensor. We conclusively show the existence of a proper segmentation algorithm from which we can extract results that are heavily correlated with TLR2 mRNA quantifications. The obtained results open possibilities for cost-effective and real-time monitoring of biomarkers with applications in embedded or labon-chip systems.
\end{abstract}

Keywords-Fluorescence microscopy, Cell monitoring, Caco-2, Toll-like receptor 2, Image segmentation, Biosensor.

\section{INTRODUCTION}

The innate immune system is an ancient, fast reacting mechanism participating in the defense of a living host against various infections. Toll-like receptors (TLR) are biological surface agents participating in this mechanism. They take an active in part recognizing exogenous molecular patterns and triggering an inflammatory response [1]. In particular, Tolllike receptor 2 and 4 (TLR2, TLR4) are well known to be upregulated by activation of their related intracellular pathways resulting from exposition to bacterial lipopolysaccharide (LPS) [2]. Quantitatively analyzing the level of expression of these receptors is a necessity, whether for deepening our knowledge about biological processes, understanding and predicting disease outcome [3], [4] or even better understanding nutrition as TLR also mediate the inflammatory action of saturated dietary fats. [5]. The current state-of-the-art in quantifying gene expressions are techniques based on reverse transcription polymerase chain reaction (RT-PCR), by which messenger $R N A$ (mRNA) coding the expression of a TLR is extracted from a cell sample, replicated and quantified. A major pro in favor of this method is its sensitivity since the PCR procedure is well controlled nowadays. However, it requires the destruction of the cell sample. As such, the measurements constitute only a snapshot of a given situation. On the other hand, immunofluorescence microscopy is a very versatile tool and can be tailored for monitoring TLR while image processing techniques are used for data extraction. In particular, one can design a complete system continuously monitoring a cell sample and its reaction to stimuli over time. This can be achieved by acquiring and processing images, then extracting data in real-time with respect to the biological processes at hand.

In this study, we monitored TLR2 expressed in Caco2 cell cultures. During the experimental step, Caco-2 cell samples were exposed to a given concentration of LPS for various periods of time, from 0 up to 24 hours. For every treatment duration, we quantified TLR2 mRNA using RT-PCR in order to obtain a reference curve. In a similar fashion, other Caco-2 cell samples were also exposed to LPS, then stained using immunofluorescence. The images were acquired using a CMOS image sensor which is able to replace CCD sensors for a range of bio-monitoring applications, including TLR monitoring [6]. The acquired images were processed using three different image segmentation schemes, which are proven to be able to localize and segment such biomarkers [7]. In this paper, we correlate the fluorescent intensities of the segmented regions with the reference curve obtained through mRNA quantification in order to demonstrate the ability of these segmentation algorithms to monitor TLR2 expression, and biomarkers in general, through conventional fluorescence microscopy. With this approach, we are able to identify the best algorithm for this job.

\section{Methodology}

\section{A. Cell samples}

For our experiment, human colon adenocarcinoma Caco2 cells (Sigma) were cultured in vitro (DMEM high glucose $4500 \mathrm{mg} / \mathrm{ml}$ with fetal bovine serum $10 \%$, L-Glutamine $4 m M$, Pen/Strep 1\%, non-essential amino acids $1 \%$ ). The cells were then exposed to a concentration of $10 \mu \mathrm{g} / \mathrm{ml}$ of LPS from Escherichia coli bacteria. Cells destined to have their TLR2 mRNA quantified through RT-PCR were treated with LPS for 1,6 and 24 hours. On the other hand, cells destined to be imaged were treated for 2, 4, 6, 12 and 24 hours. 

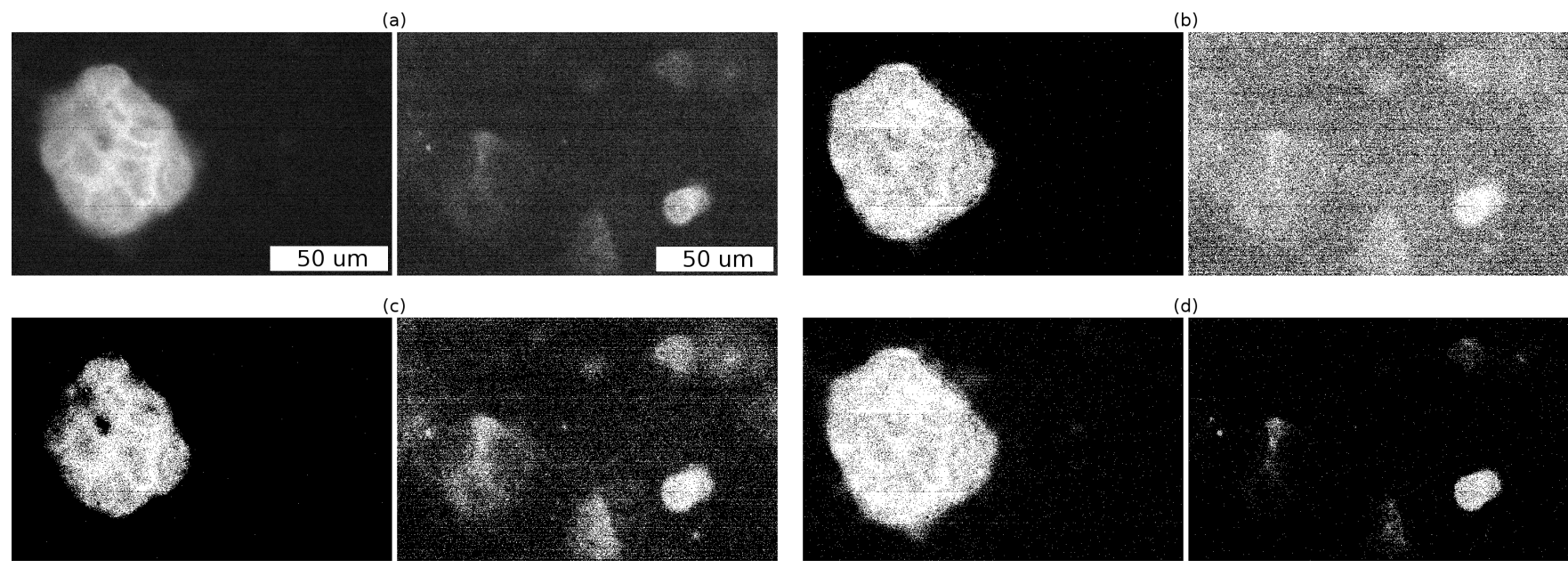

Fig. 1. (a) Sample images of fluorescent Caco-2 cells with stained TLR2 receptors acquired with the CMOS sensor after fixed pattern noise removal. Note that the constrast on these images was increased for display purposes. The left image on pannel (a) represents a packed group of cells stimulated for 24 hours with LPS. The right image however presents some isolated cells stimulated for 2 hours. Negative control images are not shown here as they all appear black. The segmentation masks obtained using Otsu (b), with Otsu2x (c) and T-point (d) were gathered after pre-processing the denoised images (a) with Top-Hat filtering.

After stimulation the cells were fixed with $3.7 \%$ formaldehyde, permeabilized with Triton-X $0.25 \%$ for 15 minutes, blocked with BSA 3\%, incubated with primary antibodies (TLR2 rabbit polyclonal $\mathrm{IgG}$ ) for 2 hours and with secondary antibodies (goat anti-rabbit IgG Texas Red) for 1 hour. A couple of negative control samples for which the LPS treatment was omitted were realized for the RT-PCR and immunofluorescence techniques, confirming a proper stimulation of the other cell samples by LPS.

\section{B. Experimental and imaging setup}

The optical fluorescence microscope used for imaging the Caco-2 cells samples is an Eclipse Ti-S inverted microscope. The objective is a Plan Fluor $(40 \times$ magnification, $N A=0.6)$, the filter set is designed for Texas Red compatible dyes $(542-576 \mathrm{~nm}$ band pass excitation filter, $595-664 \mathrm{~nm}$ pass emission filter, $533-580 \mathrm{~nm}$ reflective band and $595-800 \mathrm{~nm}$ transparent band dichroic mirror) and a $0.7 \times$ relay lens projects the image on the sensor.

The camera used for imaging the cell samples is a custom designed FPGA based system embedding a black and white CMOS sensor (MT9V032) forming a complete USB compatible camera head [8]. The major characteristics of the sensor can be found in Table I and sample images of the cell samples are gathered on the top left of Fig. 1.

\section{Image processing}

In order to be able to extract data, the various images were processed sequentially through the following three steps:

TABLE I. CHARACTERISTICS OF THE SENSOR, TAKEN FROM [6]

\begin{tabular}{lc}
\hline Designation & MT9V032 \\
\hline Sensing technology & CMOS \\
Optical format $($ in) & $1 / 3$ \\
Pixel size $(\mu m \times \mu m)$ & $6.0 \times 6.0$ \\
Pixel array size $(H \times V)$ & $752 \times 480$ \\
Pixel resolution & 10 bits \\
\hline
\end{tabular}

1) A pre-processing step, for filtering out various limiting noises and background contributions.

2) A segmentation step, where the filtered images are segmented and the regions of interest (ROIs) are divided in various connected components (CCs).

3) A data extraction step, where the average fluorescence intensity is extracted out of the CCs.

In a first step, the CMOS images were processed for fixed pattern noise (FPN) removal by subtracting a pre-computed master dark frame [8]. Images were then processed by the Top-Hat (TH) filter, which is a common approach based on mathematical morphology for enhancing the signal by removing uneven background and out-of-focus fluorescence [9].

In order to segment the pre-processed images, we employed a direct global thresholding approach for which the threshold value was automatically selected using either Otsu's algorithm [10] or the T-point algorithm [11]. Both methods are computing a threshold value based on the gray-level histogram of the pre-processed images, and do not require any other parameter. These algorithms select the threshold by maximizing a separability criterion between two classes, the background and the fluorescent pixels. The main difference between these two algorithms resides in the fact that Otsu's approach will provide best results when processing bimodal histograms, while T-point is specifically developed for handling unimodal histograms. In this study, we also considered applying Otsus algorithm recursively twice as an additional threshold selection method (named Otsu2x in the rest of this paper). Practically, this means re-applying Otsu's procedure on the initially selected upper-half of the histograms.

Further details about FPN removal, TH filtering and Otsu and T-point thresholding algorithms applied to immunofluorescence microscopy can be found in [7] and [8].

\section{D. data extraction}

We gathered 18 image datasets, each composed of 10 images taken from a single cell sample. Each set of images is 

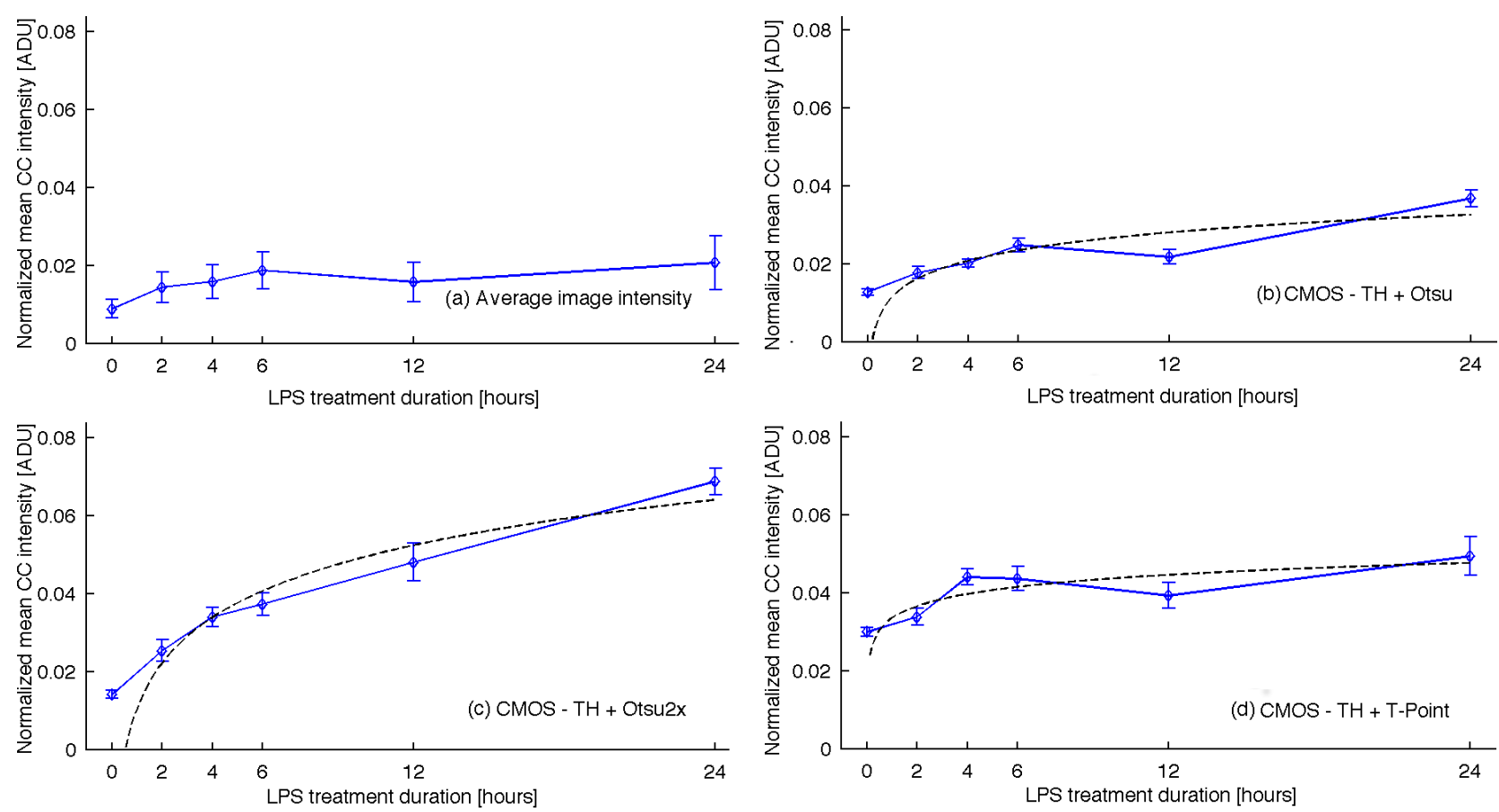

Fig. 2. Evolution of extracted fluorescence intensities linked with TRL2 expression with respect to LPS treatment duration. (a) Normalized average image intensity obtained without segmentation. (b, c, d) Normalized mean CC intensities obtained from the 3 selected image segmentation methods. The dashed line is the result of the logarithmic regression performed on the data.

characterized by:

- the employed processing method that consists of the pre-processing step followed either by Otsu, Otsu $2 x$ or T-point.

- the treatment duration of the cell sample by LPS, either 0 (negative control), 2, 4, 6, 12 or 24 hours.

After processing, the segmentation mask of every image is labeled using 4-connectivity connected component labeling. Examples of segmentation masks are depicted in Fig 1 Based on the mean pixel intensity of each $\mathrm{CC}$, we compute the mean $\mathrm{CC}$ intensity of a particular image dataset. These extracted mean CC intensities are then plotted with respect to the LPS treatment duration and compared with the mRNA measures to detect correlations.

\section{E. Statistical correlation}

For each measurement method, we are establishing correlations between the measured means. Particularly, the goal of the paper is to show that measured mean CC intensities can be correlated with measured mRNA concentrations. That is, show that these classic image-processing techniques can capture the increase of the TLR2 concentration as the LPS treatment lasts longer. To this purpose we compute Pearson's correlation coefficients (PCCs) and Spearman's ranked correlation coefficients (SRCCs) as figures of merit, in order to quantify the correlation of the measurements obtained either by Otsu, Otsu $2 x$ or T-point with the ones obtained using RT-PCR.

\section{RESULTS AND DISCUSSION}

The core or the results are presented in the various graphs gathered in Fig. 2 and Fig. 3 and the computed correlation
TABLE II. PEARSON AND SPEARMAN CORRELATION COEFFICIENTS BETWEEN THE 3 CONSIDERED METHODS AND RT-PCR

\begin{tabular}{lcccc}
\hline & Image avg. & TH + Otsu & Th + Otsu2x & TH + T-point \\
\hline Pearson $\left(\rho_{P}\right)$ & 0.813 & 0.905 & $\mathbf{0 . 9 8 9}$ & 0.805 \\
Spearman $\left(\rho_{S}\right)$ & 0.829 & 0.943 & $\mathbf{1 . 0 0 0}$ & 0.771 \\
\hline
\end{tabular}

coefficients can be found in Table II. All the measured concentrations of TLR2 mRNA (Fig. 3, solid curve) or mean intensities (Fig. 2 b, c, d) are given plus or minus the standard error (SE).

Fig. 3 (solid curve) reports the results obtained by RT-PCR where we clearly observe an increase of the TLR2 mRNA concentration as the stimulation time by LPS increases. A logarithmic regression was performed $\left(R^{2}=0.965\right)$ on the three data points for LPS treatment durations of 1,6 and 24 hours.

Fig. 2(b, c, d) presents the mean $\mathrm{CC}$ intensities obtained by processing the CMOS images using Otsu, Otsu $2 x$ and T-point after they have been corrected for the FPN. Each data point in these graphs represents the mean CC intensity computed over a data set of 10 images. As we can observe, the considered image processing methods are able to capture the evolution trend of the amount of TLR2 in the Caco-2 cells with respect to the LPS treatment duration. Table II is using the PCCs and SRCCs as figures of merit to quantify this correlation. In particular, Otsu $2 x$ appears to provide CC intensities that are highly correlated with the relative mRNA concentrations $\left(\rho_{P}=0.989 ; \rho_{S}=1.000\right)$. This result is highlighted in Fig. 3 where the measurements obtained by each method are overlapped in order to give an intuitive idea of the correlation. Another observation we can make is the relatively high correlation obtained using the average 


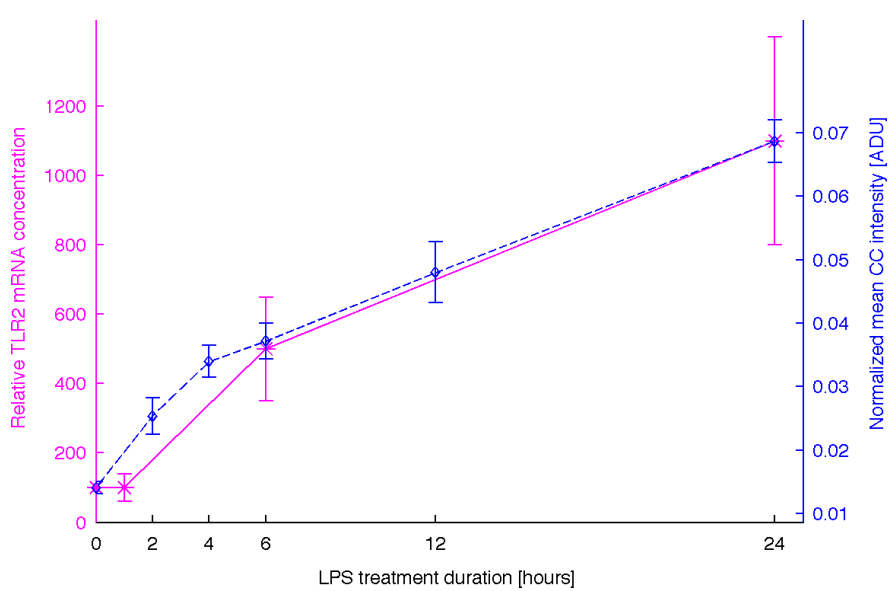

Fig. 3. Superposition of the relative TLR2 mRNA quantifications obtained by RT-PCR (solid curve, left y-axis) and the mean CC intensities obtained from $O t s u 2 x$ (dashed curve, right y-axis).

image intensity (Fig. 2a), which is the equivalent to any other cytometry technique measuring the overall fluorescence of a sample. However, the SE on the average image intensity is largely greater than that of the $\mathrm{CC}$ intensities using any segmentation method. As a result, the former is not recommended. The T-point approach provides somewhat reduced performance $\left(\rho_{P}=0.805 ; \rho_{S}=0.771\right)$, which comes from the limited performance of the CMOS imager. Notably, the low fluorescence emitted by our samples combined with the lower bit resolution and higher noise of the sensor leveled the fluorescence intensity of details from intracellular structures. The sensor is in fact acting as an averaging filter, favoring the generation of images with bimodal over unimodal histograms, in turn favoring an Otsu based approach (i.e. Otsu and Otsu2x).

A couple of further remarks need to be mentioned. During the course of our experiments, we also evaluated the correlation coefficients for the same three methods but omitting the $\mathrm{TH}$ filtering pre-processing step. All the computed coefficients were either smaller or equal to those presented here. For the sake or clarity and practicality, these results are not shown here. Second, we had to interpolate some missing datapoints in order to compute the correlation coefficients presented in section II-E. TLR2 mRNA quantifications are missing for 2, 4 and 12 hours of LPS treatment, while mean CC intensities are missing for 1 hour of LPS treatment. These missing data points were interpolated from the logarithmic regression that was performed on the measurements made for each method.

\section{CONCLUSION}

Quantitative PCR is a widely used technique for monitoring gene expression and indirectly the relative amount of a biomarker of interest on a cell sample. However, this technique requires cell lysis. Once correlated to surface receptors, even fluorimetry on entire cell samples is not capable of quantifying the biomarkers, as confirmed indirectly by Fig. 2a. In opposite we demonstrate with this paper that a correct quantification of cell surface biomarkers is possible using the right segmentation algorithm. We focus on measuring relative TLR2 concentrations in Caco- 2 cell samples by acquiring images with a lowcost CMOS image sensor and processing them using well- known image processing and segmentation techniques, namely Top-Hat filtering, T-point and Otsu's algorithm. Using these, we showed and quantified the existing correlation between the data extracted using our approach and the quantitative PCR technique. With this study, we provide a good proof that simple image processing algorithms can be used to monitor selected biomarkers from a given cell sample. Furthermore, considering this is done using CMOS technology, we have another good indicator that processing capabilities can easily be integrated on-chip, close to the sensors. This makes such systems prefect candidates for implementation in embedded systems, lab-on-chip, real-time monitoring systems and other fast, energy-efficient and cost-effective equipment.

\section{ACKNOWLEDGMENT}

This work is supported by the NutriChip project, which is financed with a grant from the Swiss Nano-Tera.ch initiative and evaluated by the Swiss National Science Foundation. This research is also partially supported by the NanoSys project, within the program ERC-2009-AdG-246810.

\section{REFERENCES}

[1] Z. Błach-Olszewska, "Innate immunity: cells, receptors, and signaling pathways." Archivum immunologiae et therapiae experimentalis, vol. 53, no. 3, pp. 245-253, 2004.

[2] J. Chen, J. N. Rao, T. Zou, L. Liu, B. S. Marasa, L. Xiao, X. Zeng, D. J. Turner, and J.-Y. Wang, "Polyamines are required for expression of toll-like receptor 2 modulating intestinal epithelial barrier integrity," American Journal of Physiology-Gastrointestinal and Liver Physiology, vol. 293, no. 3, pp. G568-G576, 2007.

[3] A. Berndt, F. Derksen, P. Venta, W. Karmaus, S. Ewart, V. YuzbasiyanGurkan, and N. Robinson, "Expression of toll-like receptor 2 mrna in bronchial epithelial cells is not induced in rao-affected horses," Equine veterinary journal, vol. 41, no. 1, pp. 76-81, 2009.

[4] K. Orihara, K. Nagata, S. Hamasaki, R. Oba, H. Hirai, S. Ishida, T. Kataoka, N. Oketani, M. Ogawa, E. Mizoguchi et al., "Timecourse of toll-like receptor 2 expression, as a predictor of recurrence in patients with bacterial infectious diseases," Clinical \& Experimental Immunology, vol. 148, no. 2, pp. 260-270, 2007.

[5] Q. Ramadan, H. Jafarpoorchekab, C. Huang, P. Silacci, S. Carrara, G. Koklü, J. Ghaye, J. Ramsden, C. Ruffert, G. Vergeres et al., "Nutrichip: nutrition analysis meets microfluidics," Lab on a Chip, vol. 13, no. 2, pp. 196-203, 2013.

[6] G. Köklü, J. Ghaye, R. Etienne-Cummings, Y. Leblebici, G. De Micheli, and S. Carrara, "Empowering low-cost cmos cameras by image processing to reach comparable results with costly ccds," BioNanoScience, 2013 , in press.

[7] J. Ghaye, M. A. Kamat, L. Corbino-Giunta, P. Silacci, G. Vergères, G. Micheli, and S. Carrara, "Image thresholding techniques for localization of sub-resolution fluorescent biomarkers," Cytometry Part A, 2013, in press.

[8] G. Köklü, J. Ghaye, R. Beuchat, G. De Micheli, Y. Leblebici, and S. Carrara, "Quantitative comparison of commercial ced and customdesigned cmos camera for biological applications," in Circuits and Systems (ISCAS), 2012 IEEE International Symposium on. IEEE, 2012, pp. 2063-2066.

[9] P. Verbeek, H. Vrooman, and L. Van Vliet, "Low-level image processing by max-min filters," Signal Processing, vol. 15, no. 3, pp. 249-258, 1988.

[10] N. Otsu et al., "A threshold selection method from gray-level histograms," IEEE Transactions on systems, Man, and Cybernetics, vol. 9, no. 1, pp. 62-66, 1979.

[11] N. Coudray, J.-L. Buessler, and J.-P. Urban, "Robust threshold estimation for images with unimodal histograms," Pattern Recognition Letters, vol. 31, no. 9, pp. 1010-1019, 2010. 